\title{
An Auxiliary-Mediated Approach for the Chemoenzymatic Synthesis of Homogenous Glycopeptides
}

\author{
Claudia Bello and Christian F.W. Becker \\ University of Vienna, Institute of Biological Chemistry, Vienna, 1090, Austria
}

\section{Introduction}

The development of effective methodologies for the production of homogeneous proteins carrying complex PTMs is essential for studying the role of the PTMs in protein function and misfunction. We established a strategy [1] for the synthesis of peptides carrying multiple and complex PTMs, focusing at first on the preparation of homogenously glycosylated peptides. The tumor marker MUC1, a protein abundantly $O$-glycosylated in its extracellular domain, was chosen as a synthetic target.

Attachment of a monodisperse PEG polymer to the $N$-terminus of our MUC1 peptides changes their solubility properties and allows sequential chemoenzymatic glycosylation of the peptides in solution and their separation from glycosyltransferases and low molecular weight components by simple techniques such as spin column gel permeation chromatography (GPC) and precipitation. This method proved to be high-yielding and versatile, as it could be coupled with native chemical ligation (NCL), thereby giving access to longer site-specifically $O$-glycosylated peptides.

Herein we present the synthesis and application of a photocleavable auxiliary, based on the 1-nitrophenyl-2-sulfanylethyl scaffold previously described by the groups of Aimoto [2] and Dawson [3], that allows attachment of the PEG polymer for efficient enzymatic glycosylation, provides the functional groups for NCL, and is cleanly removed by UV irradiation [4]. Upon photolytic removal a native glycine residue remains at the ligation site (Figure 1B).

\section{Results and Discussion}

The multistep chemical synthesis of the new auxiliary was carried out starting from vanillin and opportunely modifying the phenol moiety to accommodate the PEG polymer. In order to prepare the desired auxiliary-peptide conjugate, the MUC1 tandem repeat was synthesized by solid phase peptide synthesis (SPPS) and then modified at its $N$-terminus with an iodoacetyl group. During SPPS a $\operatorname{Thr}($ GalNAc) residue was used to introduce the first sugar of the glycan chain and have full control over the glycosylation site. $\mathrm{S}_{\mathrm{N}} 2$ reaction between the iodoacetylated peptide and the auxiliary gave the expected conjugate Aux-MUC1(Tn) in good yields (Figure 1A).

Next, we demonstrated that the PEGylated auxiliary was indeed able to facilitate the fast sequential enzymatic glycosylation of the MUC1 peptides as previously described for $N$-terminally PEGylated MUC1. Glycosylation of Aux-MUC1(Tn) with human C1GalT1 gave Aux-MUC1(T) with a single $\mathrm{T}$ antigen (Gal $\beta 1-3 \mathrm{GalNAc} \alpha$ disaccharide attached to Thr14) in excellent conversion. The incubation with a mixture of ethanol and diethyl ether at $-80^{\circ} \mathrm{C}$ induced the precipitation of the glycopeptide, which was then collected in $95 \%$ yield by centrifugation and used in the next glycosylation step without further purification. Incubation of the product with CMPNeu5Ac in the presence of recombinant ST3Gal1 allowed efficient extension of the disaccharide to the Neu5Ac $\alpha 2$ 3Gal $\beta 1-3 G a l N a c \alpha$ trisaccharide and gave Aux-MUC1(sT) with the sialyl-T antigen in 90\% yield (Figure 1A). Using similar conditions for all glycosylation reactions but by increasing times of incubation with ethanol and ether at $-80{ }^{\circ} \mathrm{C}(6-12 \mathrm{~h}$ instead of $4 \mathrm{~h})$, the sequential glycosylation procedure was simplified by omitting the GPC spin column step. As a control we have also used MUC1(Tn) without the auxiliary in this glycosylation-precipitation procedure and only $27 \%$ yield of MUC1(T) was recovered, clearly demonstrating the advantages of PEGylation.

We then coupled glycosylation and NCL reaction. A MUC1 peptide thioester (MUC1-SR) was prepared by hydrazinolysis of the MUC1 peptidyl resin and subsequent oxidation of the obtained peptide hydrazide [5]. Aux-MUC1(Tn) was converted vis sequential enzymatic glycosylation into the sialylated core 1 containing conjugate Aux-MUC1(sT), which was recovered by precipitation and directly used in a ligation reaction with the MUC1-SR thioester fragment giving the desired product MUC1-Aux-MUC1(sT) in one day and with 70\% conversion. Finally, light-induced removal of the PEGylated auxiliary was accomplished by UV irradiation of the crude ligation mixture in a water/acetonitrile mixture (Figure 1B). No starting material was detectable after 30 min of irradiation 
with an UV-A lamp and simultaneously a new peak formed corresponding to the desired product, which was isolated by HPLC purification.

To further explore the potential of this approach, the synthesis of fully unprotected conjugates comprising two glycosylated MUC1 peptides was accomplished (Figure 1C). A MUC1 peptide carrying the Tn antigen at position Thr7 (instead of Thr14) was synthesized and converted into a peptide $\alpha$-thioester. Hydrazine cleavage, deprotection, and treatment with $\mathrm{NaNO}_{2}$ and $\mathrm{MesNa}$ smoothly led to the desired MUC1- $\left(\operatorname{Tn}^{7}\right)-S R$ in $43 \%$ yield.

This peptide thioester was used in NCL with the conjugate Aux-MUC1(Tn) to give MUC1( $\left.\operatorname{Tn}^{7}\right)$ Aux-MUC1(Tn) in 35\% yield. The ligation product was efficiently used in the glycosylationprecipitation procedure leading to $\mathrm{MUC1}\left(\mathrm{T}^{7}\right)$-Aux-MUC1(T) consisting of two MUC1 tandem repeats with a $\mathrm{T}$ antigen at different positions. This much longer peptide with only one auxiliary was efficiently recovered by precipitation ( $80 \%$ recovery) under similar conditions as described above. Subsequently, it was submitted to UV irradiation at $365 \mathrm{~nm}$ for $6 \mathrm{~min}$ and the desired MUC1(T $\left.{ }^{7}\right)$-G-MUC1(T) was obtained in 53\% yield. MUC1( $\left.\mathrm{T}^{7}\right)$-Aux-MUC1(T) was also used in a further glycosylationprecipitation step leading to sialylated $\mathrm{MUC1}\left(\mathrm{sT}^{7}\right)$-Aux-MUC1(sT) (68\% recovery). UV irradiation cleanly removed the auxiliary and pure MUC1-(sT $\left.{ }^{7}\right)$-G-MUC1(sT) was obtained in $12 \%$ yield after HPLC purification (Figure 1C). These results demonstrate the power of this approach for the chemoenzymatic synthesis of glycosylated peptides and its combination with NCL to obtain larger polypeptides with different but specific glycosylation patterns.

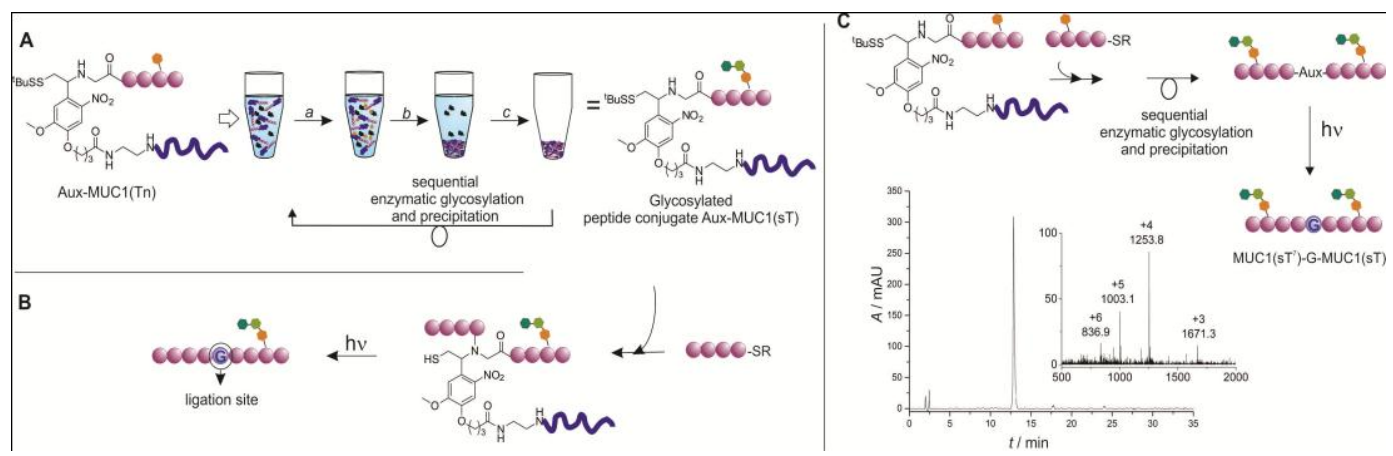

Fig. 1. Auxiliary-mediated enzymatic glycosylation (A) and ligation approach (B). HPLC chromatogram and ESI-MS spectrum of the native Mucin1 analog $M U C 1\left(s T^{7}\right)-G-M U C 1(s T)(C)$.

\section{Acknowledgments}

We thank the MS center of the Department of Chemistry at the University of Vienna for help with MS analysis of a variety of compounds, and M. Arndt, L. Mairhofer, and J. Cotter for help with laser experiments. The Alexander von Humboldt foundation is kindly acknowledged for financial support to C.B.

\section{References}

1. Bello, C., Farbiarz, K., Moeller, J.F., Becker, C.F.W., Schwientek, T. Chem. Sci. 5, 1634-1641 (2014), http://dx.doi.org/10.1039/C3SC52641K

2. Kawakami, T., Aimoto, S. Tetrahedron Lett. 44, 6059-6061 (2003), http://dx.doi.org/10.1016/S00404039(03)01463-1

3. Marinzi, C., Offer, J., Longhi, R., Dawson, P.E. Bioorg. Med. Chem. 12, 2749-2757 (2004), http://dx.doi.org/10.1016/i.bmc.2004.02.039

4. Bello, C., Wang, S., Meng, L., Moremen, K.W., Becker, C.F.W. Angew. Chem. Int. Ed. 54, 7711-7715

(2015), http://dx.doi.org/10.1002/anie.201501517

5. Bello, C., Kikul, F., Becker, C.F.W. J. Pept. Sci. 21, 201-207 (2015), http://dx.doi.org/10.1002/psc.2747 